\title{
LA DIMENSIONE REGIONALE DEL TEATRO ITALIANO: LA SICILIANITÀ NAZIONALE E L'ALTERNATIVA PIRANDELLIANA
}

\section{Maria Elena Santuccio}

\begin{abstract}
Riassunto: Il discorso nazionale sulla meridionalità diffonde attraverso il teatro verista un modello di sicilianità passionale $\mathrm{e}$ tradizionalista. Questo modello integra la sicilianità all'italianità ed è presentato come 'essenziale' attraverso la drammaturgia del vero. Utilizzando la ripetizione straniante di elementi strutturali del teatro verista Pirandello contesta l'immagine della Sicilia come luogo di passione e baluardo della tradizione.
\end{abstract}

Le scene teatrali tra Ottocento e Novecento costituiscono uno dei principali circuiti del discorso nazionale sulla meridionalità e sulla sicilianità. Le immagini della meridionalità siciliana, veicolate dal teatro ed in particolare dalla produzione verista, rispondono alla necessità di integrare la sicilianità all'italianità e si muovono tra le tipizzazioni complementari della primitività passionale e violenta e della primitività come genuinità. La Sicilia viene caratterizzata come luogo 'diverso' di passioni irrefrenabili, ma, allo stesso tempo, radicalmente italiano, tanto da rappresentare una sorta di baluardo in difesa del valore 'tradizionale' della rispettabilità borghese mascherata da onore 'arcaico'.

Dopo aver definito la funzione del teatro regionale nel processo di costruzione della nazione e nella diffusione dei valori borghesi della rispettabilità, della gerarchia e della famiglia, discuteremo l'elaborazione della sicilianità nel contesto della pratica discorsiva positivista come 'essenza' di passionalità e tradizionalismo. L'analisi delle strutture drammatiche rivela come nella produzione teatrale verista mimesi e catarsi sorreggano sia la visione essenzialista della sicilianità che l'intento moralista. Il teatro verista siciliano, che comprende opere quali I mafiusi (1863) di Giuseppe Rizzotto e Gaspare Mosca, La zolfara (1888) di Giuseppe Giusti Sinopoli, Cavalleria rusticana (1884) e La lupa (1896) di Giovanni Verga, Malia (1895) di Luigi Capuana, raffigura un universo popolare passionale e violento ma fondamentalmente sano.

Questo universo viene descritto come 'vero', la cura del dettaglio realistico fissa con precisione 'scientifica' una 'natura' siciliana. Nella descrizio- 
ne di tale 'natura' rispettabilità e religione svolgono un ruolo fondamentale. La rispettabilità viene infatti presentata come insita nella sicilianità in veste di arcaico sentimento religioso. La struttura drammatica delle opere veriste è di tipo pedagogico ed è centrata sul carattere del personaggio passionale che, a causa della propria 'incontinenza', non osserva le regole della comunità. La trasgressione viene solitamente punita per mezzo del delitto d'onore che ristabilisce la naturale armonia, confermando così la 'sacralitä' dei valori violati.

Il modello di primitività 'naturale' viene rifiutato dalla cultura siciliana d'opposizione che indaga sul rapporto tra la Sicilia e il Nuovo Regno, fuori dall'ambito del determinismo biologico e razziale. Vedremo come il teatro in dialetto di Pirandello partecipi ai discorsi della contro-cultura regionale contrastando la definizione dell'identità siciliana nazionale elaborata dalla produzione verista. Il raddoppiamento differenziante pirandelliano costituisce un meccanismo epico che interviene contro l'illusionismo drammatico per rovesciare parodicamente il binomio essenza-siciliana/nazionalismo. Lepicizzazione introduce un rapporto soggetto-oggetto che relativizza il 'documento umano', l'assolutezza 'scientifica' della scena verista'

Nelle opere 'siciliane' di Pirandello, 'A birritta cu' i ciancianeddi (1917), Liolà (1916), 'A patenti (1918), Pensaci Giacuminu! (1916), troviamo la ripetizione straniante di elementi strutturali del teatro verista quali la passione, il delitto d'onore, la religione, il matrimonio. Alla passione-pazzia, che innesca la trasgressione e che si manifesta in quanto patologia magicodemoniaca del carattere (Cavalleria rusticana, La lupa, Malia), si contrappone la pazzia come costruzione ('A birritta cu' i ciancianeddi) o la magia come finzione attivamente e gioiosamente praticata ('A patenti); alla violenza passionale che porta alla vendetta da parte del marito/fidanzato per difendere l'onore (Cavalleria rusticana, I mafiusi, La zolfara) si sostituisce l'impunità dell'azione fecondatrice (Liolà) o il triangolo amoroso allegramente vissuto dal marito (Pensaci Giacuminu.); alla religione come patrimonio morale atavico della comunità (I mafiusi, Cavalleria rusticana, La lupa, Malia) una religione 'personalizzata', da rielaborare a seconda delle circostanze per legittimare determinate azioni (Liolà); al matrimonio riparatore (Malia) o impossibile (La lupa) il matrimonio-burla per protestare

${ }^{1}$ Per il rapporto tra letteratura realista e forme di normalizzazione si veda il saggio di Leo Bersani, "Le réalisme et la peur du désir". La differenza fra teatro drammatico tradizionale, 'aristotelico-naturalistico', e teatro epico o 'narrativo' è analizzata da Bertolt Brecht in Schriften zum Theater. Sui meccanismi di epicizzazione del dramma moderno è fondamentale lo studio di Peter Szondi Teoria del dramma moderno. 
contro lo Stato (Pensaci Giacuminu!). L'analisi del teatro siciliano permette infine di allargare il quadro del regionalismo contro-culturale di Pirandello, solitamente circoscritto al romanzo storico I vecchi e i giovani (1913).

\section{La costruzione della nazione: regione e cultura popolare nel discorso sull'i- dentità italiana}

In Italia il processo di formazione 'immaginativa' della nazione fu segnato da discorsi che fissarono un nucleo egemonico, il Nord, progredito e civile, ed una zona marginale, il Sud, arretrato e primitivo ${ }^{2}$. Pratiche discorsive di orientamento imperialista, orientalista e razzista diffusero la convinzione che i meridionali possedessero caratteristiche essenziali opposte a quelle dei settentrionali. Passionali e indisciplinati, i meridionali erano incapaci di sviluppare quella educazione civica che nel Nord rendeva possibile la formazione di una società avanzata.

Eric Hobsbawm ha esaminato il fenomeno del 'nation-making' che caratterizzò la seconda metà dell'Ottocento e ha indicato come i progetti di 'costruzione della nazione' fossero legati al principio fondamentale di unificazione. La nazione veniva considerata la cellula 'naturale' di sviluppo della moderna società liberale, progressista e borghese. Lo Stato-nazione doveva essere indipendente e sviluppare un'organizzazione statale, un'economia e una forza militare proprie. Per essere indipendente la nazione doveva essere di una certa grandezza. Bisognava quindi 'unificare': i popoli piccoli e considerati arretrati non avevano carattere di 'vere' nazioni e dovevano essere integrati negli stati-nazione che erano dotati di vitalità propria. Le nazioni grandi, le 'progredite', erano destinate a uscire vincitrici dalla lotta per l'esistenza 3 .

Il discorso nazionalista si articolò inoltre attraverso il confronto tra le varie nazioni. Già a partire dalla seconda metà del Settecento si svilupparono delle tendenze 'orientaliste' che delinearono un'opposizione tra Nord e Sud d'Europa in termini di sviluppo e arretratezza ${ }^{4}$. Nelson Moe ha evi-

2 Mi riferisco al concetto di nazione come 'comunità immaginata'. Benedict Anderson ha sottolineato che le nazioni non sono solo formazioni politiche ma 'imagined communities', cioè sistemi di rappresentazione culturale. Secondo Anderson l'identità nazionale viene continuamente riprodotta attraverso l'attività discorsiva.

3 Hobsbawm, Il trionfo della borghesia: 1848-1875.

${ }^{4}$ Edward Said ha dimostrato che entità culturali e geografiche come l'Oriente' sono specifiche costruzioni discorsive. L'orientalismo comprende una serie di discorsi di potere occidentali. Attraverso questi discorsi si è costruito un Oriente 
denziato il fatto che l'Italia veniva considerata un paese 'meridionale', a dispetto delle élite liberali risorgimentali e post-unitarie che miravano invece ad uno Stato italiano da collocare a fianco degli stati europei più avanzati: Inghilterra, Francia e Germania. Dovendo fare dell'Italia una nazione 'settentrionale', la parte meridionale del paese fu identificata come diversa, distante dalla più progredita civiltà europea occidentale e più vicina all'Africa e all'Oriente. Al contrario, l'identità settentrionale italiana si consolidò come moralmente, culturalmente e tecnicamente superiore al Sud'. La definizione dei meridionali in quanto 'diversi', primitivi e incivili, trovò un appiglio 'scientifico' nelle ricerche antropologiche di Cesare Lombroso, Giuseppe Sergi, Enrico Ferri e Alfredo Niceforo secondo i quali la razza aveva un ruolo fondamentale nel determinare il comportamento sociale. I 'darwinisti' italiani stabilirono l'inferiorità razziale dei meridionali attraverso misurazioni del cranio ed altri criteri pseudoscientifici, fornendo così una giustificazione alla durissima repressione dei disordini sociali nel Sud e specialmente in Sicilia.

Se il meridione veniva qualificato come 'diverso', primitivo rispetto al modello di società capitalistico-industriale a cui l'Italia liberale aspirava, per costruire la nazione occorreva trovare nella diversità del sud elementi di italianità. La primitività meridionale fu quindi integrata all'italianità in quanto primigenia autenticità che legittimava i valori morali nazionali. Il teatro regionale svolge questa funzione di aggregazione e in genere, piuttosto che essere espressione di una realtà locale o popolare, possiede un carattere 'unitario'.

La 'dimensione regionale' del teatro italiano è ben caratterizzata dall'esistenza tra la fine dell'Ottocento e gli inizi del Novecento di una produzione dialettale di ambientazione locale e popolare o piccolo-borghese ${ }^{6}$. Si tratta di una proposta teatrale che suscitò grande interesse, basti pensare non solo al teatro verghiano e verista siciliano, ma anche al successo di opere appartenenti ad altri ambiti regionali. Ricordiamo, ad esempio, Miseria e nobiltà (1888) di Edoardo Scarpetta, Assunta Spina (1909) e 'O mese mariano (1900) di Salvatore Di Giacomo per il teatro napoletano, Le miserie di Monssù Travet (1863) di Vittorio Bersezio per il teatro piemon-

in modo tale da riprodurre l'egemonia dell'Occidente. L'orientalismo comprende tutta una serie di idee centrate sulla superiorità occidentale, sul razzismo e sull'imperialismo.

5 Moe, The View from Vesuvius, 13.

${ }^{6}$ L'utilizzazione del dialetto è molto diversificata in forme più o meno ibride. Dal dialetto milanese puro di Bertolazzi all'italiano con colore locale siciliano di Verga. 
tese, La povera gent (1893) di Carlo Bertolazzi per il teatro milanese e $L a$ famegia del santolo (1891) di Giacinto Gallina per il teatro veneto. Questi sono solo alcuni esempi di un'attività teatrale molto varia. La più generica definizione di teatro dialettal-regionale nasconde in realtà una molteplicità di rapporti con il teatro 'nazionale' in lingua. Abbiamo quindi opere che nascono in dialetto e poi vengono tradotte per le compagnie in lingua o, viceversa, ci sono rifacimenti dialettali e adattamenti regionali di opere in lingua e persino di successi del teatro straniero.

La produzione regionale in dialetto si intreccia con quella in lingua ed ha una funzione 'unificante'. Questo teatro cerca di inglobare la 'cultura regionale' all'interno di quella nazionale. Nella prefazione al primo volume del suo teatro siciliano (1911), Luigi Capuana si dimostra convinto sostenitore della scena dialettale. A quanti vorrebbero abolire tutti i teatri dialettali, almeno per un secolo, perché gli italiani pensino a creare opere di qualità in lingua italiana, il Capuana risponde: "Come se si trattasse di dialetti ostrogoti! Come se i personaggi veneziani, fiorentini, napoletani, siciliani non fossero forse più italiani di quelli del teatro non dialettale, perché più sinceri, al pari degli attori che li rappresentano!" E aggiunge: "Io credo che bisogna passare per i teatri dialettali, se si vuole davvero arrivare al teatro nazionale italiano"7. Vittorio Bersezio, dopo il successo di Monssù Travet in piemontese, scrive una versione in italiano, sostenendo che "la commedia non fu esclusivamente torinese, ma di costumi generali e perciò la si volle tradotta" 8 .

La produzione dialettal-popolare, considerata documento di vita locale, fu incoraggiata per la sua funzione moralizzatrice nei confronti degli spettatori popolari e borghesi. Come ha osservato Silvana Monti, la difesa del teatro dialettale quale strumento culturale e civile ritorna spesso nelle pagine dei giornali contemporanei. Le produzioni dialettali dovevano "educare il popolo nella morale e mantenerlo nella giustezza del sentimento politico". Esse, inoltre, venivano apprezzate in quanto presentavano personaggi "naturali" che davano "un'idea molto vera dei costumi sociali del popolo".

Il teatro si inserisce in quel movimento di 'scoperta' della 'cultura popolare' che fu parte integrante dell'emergere del nazionalismo europeo. Già a partire dalle opere di Johann Gottfried Herder, nella seconda metà del Settecento, troviamo l'idea ricorrente della cultura popolare come materia grezza, come natura nella quale la cultura della nazione può crescere e svilupparsi. Anche nel corso dell'Ottocento la 'cultura popolare'

\footnotetext{
7 Capuana, Teatro dialettale siciliano, 72.

8 Bersezio, Le miserie e prosperità del signor Travetti, 18.

9 Monti, Il teatro realista della nuova Italia, 50.
} 
continuò ad attirare la costante attenzione di letterati, studiosi di folklore e uomini politici. Si raccolsero non solo canti, proverbi, fiabe, ma anche dati sulle condizioni di vita e di lavoro delle classi subalterne, esplorate attraverso le inchieste promosse dopo l'unificazione.

L'interesse per il vernacolo e per la vita regional-popolare rientra nel processo di costruzione della nazione. Nell'ambito della classi subalterne delle diverse regioni si potevano ritrovare elementi di una cultura comune. Il popolo viene presentato quale conservatore dei sentimenti naturali e della tradizione. In una recensione a una raccolta di proverbi popolari uscita nel 1853 e poi ripubblicata nel 1888 il giornalista e critico Carlo Tenca affermava: "nessuno più del popolo è tenace delle consuetudini della famiglia, e serba, a traverso la licenza dei costumi e la corruzione cittadina, quel senso di divozione ai vincoli dell'affetto ed all'autorità dei vecchi padri, che è tanta parte di pubblica moralită" 10 .

Il teatro regionale svolge una funzione di coesione, diffondendo una visione 'conciliante' e non problematica del popolo. Attraverso il teatro si sviluppa un discorso di superamento dei confini immaginativi tra classi diverse, tra nazione e regioni, tra arte e folclore. Gli elementi unificanti sono i valori borghesi dell'onore, del decoro, della famiglia, della moderatezza e del rispetto dell'ordine gerarchico.

\section{La Sicilia genius loci della nazione}

Il teatro verista siciliano partecipa al processo di costruzione immaginativa attraverso il quale la Sicilia viene collocata all'interno del nuovo Stato italiano. Se il Sud fu definito proprio tramite il confronto 'negativo' con il Nord, la diversità del meridione doveva in qualche modo essere resa 'interna' per potere immaginare un'Italia integrata. Bisognava insomma trovare elementi di italianità nella meridionalità. Accanto all'immagine della primitività istintiva e passionale si sviluppò quella del Sud come riserva di tradizioni popolari e di certezze religiose minacciate dalla modernizzazione.

La visione verista della Sicilia si inserisce tra le pratiche discorsive che modellarono l'immagine del Sud alla fine dell'Ottocento. John Dickie ha fatto notare come in questo periodo si assista ad una proliferazione di rappresentazioni del meridione. Soprattutto nella rivista Illustrazione italiana, organo che svolse un'importantissima funzione 'nazionale', la cultura meridionale trovò ampio spazio. La rivista cercò di sviluppare un'estetica patriottica attraverso l'arte e la letteratura. La nozione di una tradizione

10 Ceserani, Il Materiale e l'immaginario, 172. 
artistica e letteraria italiana rimase una fondamentale garanzia della solidità della nazione contro i conflitti politici. Il teatro faceva parte di questa tradizione comune, si parlava infatti di "nostro amore per il teatro"11. Il meridione che emerge dalle pagine dell'Illustrazione è il luogo per eccellenza della cultura popolare, della tradizione, del mito e del pittoresco. La meridionalità viene presentata come esotica e allo stesso tempo familiare, in posizione intermedia tra barbarie e civiltà ${ }^{12}$.

Proprio questa immagine, fissata dalla carica gestuale e mimica degli attori Giovanni Grasso e Mimì Aguglia ${ }^{13}$, dominerà nel teatro verista siciliano, contribuendo alla definizione di una 'essenza siciliana' nazionale che, come dimostreremo più avanti, sarà poi contestata da Pirandello agli inizi del Novecento ${ }^{14}$.

Facevano parte del repertorio ${ }^{15}$ dei due attori siciliani e delle loro com-

11 Dickie, Darkest Italy, 88.

12 Dickie, Darkest Italy, 83.

13 Nel 1903 Gabriele D’Annunzio, dopo aver visto al Manzoni di Milano La zolfara di Sinopoli, rappresentata da Giovanni Grasso, giudicò l'attore "meraviglioso strumento d'arte", espressione potente dell'"anima originale della stirpe". Sipala, "Una cosa nuova che la chiamavano sciopero", 858. Sul 'verismo' dello stile recitativo di Grasso si veda il saggio di Enzo Zappulla, "Giovanni Grasso, magnifico attore tragico siciliano".

14 Sarah Zappulla Muscarà nell'introduzione a Tutto il teatro in dialetto di Pirandello ha fatto notare il legame tra il teatro antinaturalistico pirandelliano e lo stile recitativo dell'attore Angelo Musco: "Sarà, invece, Angelo Musco a segnare con gli accenti del comico lo spartiacque ideale tra il modello interpretativo e testuale del teatro verista, portato al successo da Grasso, ed i fermenti di nuovi moduli espressivi nei quali si avvertivano i primi segni di mise en scène umoristica (...) Lontane dai consueti clichés di un teatro sanguinario o macchiettistico, stupefacenti tematiche si facevano via via strada, non senza difficoltà, tra un pubblico non ancora aduso a soluzioni così antitradizionalistiche. E l'arte muschiana, che mostrava talora una complessa natura drammatica, una sensibilità più raffinata, bene si accordava con il verticalismo del corrosivo teatro pirandelliano" (vi-x). Su Angelo Musco si veda inoltre la raccolta di saggi a cura di Enzo Zappulla, Angelo Musco e il teatro del suo tempo.

15 Anche opere originariamente in lingua potevano essere tradotte in siciliano ed entrare a far parte del repertorio dialettale. È interessante a tal proposito il caso di La morte civile (1861) di Paolo Giacometti. L'attore Ermete Zacconi spiegò che l'autore volle come protagonista un siciliano, e "propriamente nato ai piedi dell'Etna", perché "egli aveva bisogno di una di quelle nature atte sia a uccidere per causa passionale sia ad uccidersi per magnanimità di sacrificio". Ferrone, 
pagnie I mafiusi (1863) di Giuseppe Rizzotto e Gaspare Mosca, La zolfara (1888) di Giuseppe Giusti Sinopoli, Cavalleria rusticana (1884) e La lupa (1896) di Giovanni Verga, Malia (1895) di Luigi Capuana. In queste opere l'universo siciliano deve rivelarsi come 'vero', bisognava fare conoscere la 'vera essenza'16 della Sicilia e offrire al pubblico un 'documento umano'.

I personaggi sono caratterizzati da una forte passionalità, in consonanza con una visione 'primitiva' della Sicilia. I siciliani sono italiani in modo 'primordiale', la loro passionalità è inserita in un quadro di valori tradizionali che coincidono con quelli nazionali: la famiglia e la religione. La produzione verista si muove nel solco di un'affermazione della radice cristiana dell'italianità. La dottrina sociale della Chiesa basata sull'accettazione del proprio stato, sull'armonia tra le classi, sul rispetto della gerarchia forniva infatti un forte elemento ideologico di coesione nazionale di fronte alle agitazioni sociali. Negli anni ' 80 il crescente disordine sociale convinse l'élite politica della necessità di rafforzare l'autorità dello Stato attraverso una riconciliazione con la Chiesa. Ciò avrebbe dato allo Stato legittimità e fornito un potente scudo ideologico contro l'avanzata del socialismo ${ }^{17}$.

Teatro italiano, 439. L'associazione del siciliano con la forza naturale del vulcano ci dà un'idea di come si sviluppi nella pratica discorsiva un'essenza, una 'natura' siciliana. Corrado, il vulcanico eroe della Morte civile, è fisiologicamente passionale: può dar sfogo alla violenza del suo temperamento ed uccidere, ma può anche dirigere la passionalità a buon fine e suicidarsi per magnanimità.

16 Occorre notare che i critici a loro volta hanno contribuito alla visione essenzialista della Sicilia e dei siciliani. Abbiamo una storia che si trasforma in natura, una sicilianità che esiste fuori delle pratiche discorsive. Vediamo, ad esempio, alcuni giudizi a proposito del teatro verista siciliano: Federico De Roberto definiva il rituale del morso di Turiddu che accetta il duello con Alfio in Cavalleria rusticana di Verga come "espressione dell'anima siciliana, rivelatrice dell'ampiezza dei suoi gesti e dell'impeto delle sue passioni". (Verga, Teatro, 40). Anna Barsotti individua in Cavalleria rusticana un "regionalistico concetto dell'onore" e "la tendenza dei popoli meridionali a farsi giustizia da sé", (Barsotti, Verga drammaturgo, 41), inoltre, nei Mafiusi di Rizzotto-Mosca, riscontra un "atteggiamento particolare del maschio siciliano verso la femmina" e un "valore particolare attribuito dalla mentalità locale all'onore della donna",( 43); più di recente Daniela Bini a proposito di Cavalleria rusticana parla di "natura dicotomica della donna siciliana (donna di piacere da un lato /vergine e madre dall'altro) radicata nella psiche e nella cultura siciliana". ("dichotomy erotic woman/virgin mother, so ingrained in the Sicilian psyche and culture"). (Bini, "Cavalleria Rusticana from Verga and Mascagni to Zeffirelli”, 96).

17 Duggan, 96. 
Le rappresentazioni veriste della sicilianità si intrecciano con la necessità di disciplinare, controllare e unificare il corpo sociale. Occorreva legarsi alle narrazioni universalistiche nazionali. Per Verga la patria occupava una posizione prioritaria nella 'missione umanitaria' dell'intellettuale italiano. Quando Edoardo Boutet contestò i veristi perché non si impegnavano a favore delle classi popolari ${ }^{18}$, Verga, nell'introduzione alla versione narrativa di Dal tuo al mio, dava una risposta alle critiche: "se il teatro e la novella, col descrivere la vita qual è compiono una missione umanitaria, io ho fatto la mia parte in pro degli umili e dei diseredati da un pezzo, senza bisogno di predicar l'odio e di negare la patria in nome dell'umanità" 19 .

Ma esaminiamo più da vicino alcune opere veriste. La commedia $I$ mafiusi, lavoro d'incerta origine attribuito a Gaspare Mosca e Giuseppe Rizzotto, viene considerata la prima opera del verismo teatrale siciliano. Fu rappresentata per la prima volta nel 1863 al Piccolo Teatro Sant'Anna di Palermo, teatro che ospitava in quell'epoca il repertorio popolare.

La struttura di I Mafiusi indica in modo emblematico l'integrazione' dell'elemento 'locale-popolare' con quello nazionale. Originariamente il testo dell'opera comprendeva solo due atti caratterizzati dal susseguirsi di scene che ritraevano la vita dei detenuti nelle carceri di Palermo. Abbiamo il pagamento del pizzo da parte dei nuovi arrivati, il gioco a carte, le scommesse, le piccole truffe, le visite dei familiari, lo smascheramento della spia della polizia. L'andamento non era lineare e i personaggi non seguivano il percorso di formazione tipico del teatro borghese. L'opera, troppo 'documentaria' e non 'pedagogica', suscitò contrasti e la critica indicò quelle scene come fonte di corruzione morale. In seguito a tali accuse, furono aggiunti il primo e il quarto atto che, come vedremo, dotarono il lavoro di una struttura pedagogica e di una dimostrazione morale. Il lavoro riveduto piacque al sindaco di Palermo, il marchese Di Rudinì, che lodò il Rizzotto e lo incoraggiò a proseguire a scrivere per il teatro siciliano: "Ella s'impegna a scrivere ancora nel lodevole intento di cooperare all'incivilimento del nostro paese. Vada avanti signor Rizzotto (...) e ponga mente che una buona lezione sulla scena vale cento di quelle che si danno in iscuola,

18 Edoardo Boutet, uno dei più illustri critici teatrali dell'epoca, scrisse articoli per vari giornali e riviste fra cui Il Corriere di Roma, il Don Chisciotte, l'Avanti, la Nuova Antologia, la Rivista d'Italia. Nel 1894 in un articolo anti-verista pubblicato sul Don Chisciotte si schierò contro "il folclorismo amabile e sentimentale" e contro "i compari Turiddu e compari Alfio, e i morsetti all'orecchio e le male Pasque a te e a me". Ferrone, Teatro italiano, 235.

19 Verga, Tutte le novelle, 309. 
perché al teatro si va vecchi e fanciulli e sempre volentieri, mentre alla scuola si va per lo più condotti per mano dal babbo e dalla mamma"20.

Il 17 dicembre 1875 la giunta municipale di Palermo assegnò al Rizzotto la somma di cinquecento lire per premiare "l'impegno di creare nel nostro dialetto la commedia popolare, per l'educazione delle masse"21.

Ma vediamo cosa viene aggiunto: ad uno dei personaggi dei due atti originari, Gioachino Funciazza, si dà la funzione di eroe e gli si fa seguire un processo lineare di formazione, dall'omicidio alla vita onesta. Il primo atto serve per chiarire che il Funciazza va in prigione per aver ammazzato in un attacco d'ira il presunto amante della moglie. Purtroppo non è riuscito a controllarsi, ma la causa era giusta: voleva difendere gli affetti domestici, la famiglia. Del resto risulta piuttosto significativo il fatto che gli verrà concessa l'attenuante della 'ragione d'onore'. Nel quarto atto scopriamo che, dopo aver scontato la pena di soli cinque anni, il Funciazza ha aperto una bottega di calzolaio e lavora onestamente. Ad un certo punto, riceve la visita dei suoi ex-compagni di carcere i quali cercano di convincerlo a partecipare ad una truffa. Sebbene poverissimo, Gioachino non cede alla proposta disonesta. Il motivo, dice, è che il carcere gli fa paura: la sua assenza durante la prigionia ha provocato la morte della madre e per poco anche quella della moglie e del figlio. È come se Gioachino avesse sconvolto l'ordine naturale e fosse stato punito dall'intervento divino. Apprendiamo che la madre si era ammalata il giorno di Pasqua ed era morta senza benedirlo. Allo stesso tempo, però, il Funciazza viene 'redento' dal dolore per la morte della madre e per gli stenti di moglie e figlio. Egli 'risorge' ad una vita nuova e onesta grazie ad una sofferenza che lui stesso definisce 'martirio'. Il ripetersi dei riferimenti religiosi colloca il siciliano in una dimensione di moralità assoluta ed immutabile. Come avremo modo via via di constatare, la religiosità della comunità siciliana è una costante delle opere veriste.

Con la revisione I mafiusi vengono adattati alle norme pedagogiche del teatro borghese. Si concentra l'attenzione sul nucleo familiare e si presentano i 'mali' che minacciano l'armonia naturale' di tale nucleo. Alla colpa, alla trasgressione contro la stabilità familiare segue inevitabilmente l'espiazione. La rigenerazione proviene sempre dal privato: riscattato dalla forza salvifica della famiglia, il Funciazza, ormai onesto lavoratore, ristabilisce l'ordine naturale e divino. Nella battuta conclusiva l'eroe si augura che i

\footnotetext{
20 Barbina, Teatro dialettale siciliano, 45.

21 Barbina, 49.
} 
compagni seguano il suo esempio e si trovino un lavoro onesto, perché "il lavoro è l'unico mezzo che può render felice e contento l'individuo, la famiglia e formare la grandezza d'una intera nazione"22. Abbiamo una concezione del lavoro come istinto che ha nella natura stessa la sua giustificazione e lega insieme in maniera non problematica e 'naturale' individuo, famiglia, società e religione.

Al centro di Cavalleria rusticana del Verga si trova il solito triangolo del teatro borghese collocato in un ambiente rurale. La ruralità rinforza ideologicamente i valori nazionali, il matrimonio, la famiglia e l'onore. Attraverso la ruralità tali valori sono mostrati come appartenenti ad una dimensione arcaica, elementare, e vengono così fissati come eterni e naturali.

Questo è l'antefatto rivelato nei dialoghi dei personaggi: Turiddu torna a casa dopo il servizio militare e trova che Lola, con cui amoreggiava prima di partire, nel frattempo ha sposato Alfio. Indispettito, Turiddu comincia a corteggiare Santuzza. Lola, spinta dalla gelosia verso Santuzza, si riavvicina all'antico fidanzato e ne diventa l'amante. L'azione del dramma si concentra invece nel momento della catastrofe finale in cui scoppia la tensione passionale. Il giorno di Pasqua Santuzza, che probabilmente è incinta di Turiddu, fa una scenata di gelosia al giovane. Da lui respinta, si vendica informando Alfio della tresca di Lola con Turiddu. Alfio sfida Turiddu a duello, Turiddu rimane ucciso.

La violazione della sacralità del matrimonio causa la tragedia. Il tempo della festa religiosa, la Pasqua, coincide con la punizione dei peccatori. La forza motrice del dramma è la passione incontenibile che accomuna tutti i protagonisti. La catastrofe è preparata coralmente da un gioco di sentimenti 'a catena': Turiddu, mosso dal risentimento nei confronti di Lola corteggia Santuzza; Lola, gelosa di Santuzza, intraprende una relazione con Turiddu; Santuzza, gelosa di Lola, denuncia l'adulterio; Alfio, per vendicarsi contro chi gli ha 'messo il coltello nel cuore', sfida e uccide l'amante della moglie. Non c'è quindi da stupirsi se Giovanni Grasso nei suoi arrangiamenti di Cavalleria rusticana esagerava fortemente gli aspetti passionali. Considerato il predominio della carica emozionale nelle motivazioni dei personaggi, l'opera incoraggiava tali interpretazioni ${ }^{23}$.

D'altro canto, se esaminiamo più da vicino i motivi dell'azione, vediamo che la violenza passionale è accompagnata dal desiderio di difendere la propria rispettabilità, il proprio onore. Prendiamo ad esempio la scena che

22 Barbina, 139.

23 Anche Verga criticò gli allestimenti 'bestiali' di Giovanni Grasso durante il periodo delle sue recite parigine nelle stagioni 1907-1909. 
provoca la catastrofe finale, cioè la denucia dell'adulterio. Santuzza prega Turiddu di non andare in chiesa a raggiungere Lola. Oltre che una trasgressione delle norme religiose (Turiddu continuerebbe a commettere peccato se seguisse Lola in Chiesa), sarebbe un affronto a lei di fronte alla comunità (Turiddu è promesso a Santuzza e dovrebbe andare in chiesa con lei e non con Lola). Santuzza racconta tutto ad Alfio per reagire all'offesa contro il proprio onore. Anche Turiddu agisce con onore. Egli riconosce la sua colpevolezza e dice di essere pronto a morire, a farsi ammazzare da Alfio e ad espiare per la 'legge dell'onore'. La scelta da parte di Alfio del duello, lungi dall'essere espressione di aggressività incontrollata, rappresenta invece una forma di controllo dello spontaneo, dell'istinto. Il duello è simbolo di un comportamento 'civile' arcaico. Esso faceva parte dell'etichetta comportamentale della classe nobiliare. Non a caso il titolo del dramma fa riferimento alla cavalleria.

Secondo il discorso 'nazionale' del Verga, nonostante la loro genetica passionalità, i siciliani hanno una fondamentale 'sanità morale che è garantita dalla tradizione. Il personaggio ha un carattere essenzialmente onesto e ha interiorizzato le leggi della comunità, purtroppo, però, la passionalità incontrollata lo porta alla trasgressione. La comunità è una società omogenea e compatta inserita nel tempo rituale ed immutabile della ricorrenza religiosa. Le voci dei personaggi sono espressione di una saggezza atavica e vengono unificate attraverso il ricorso alla forma impersonale, ai proverbi e agli aforismi.

Questo universo popolare è presentato come reale. Il pubblico deve identificarsi con i personaggi, deve commuoversi ed imparare una lezione morale. Sappiamo che per la prima dell'opera si curarono i dettagli nella scenografia e nei costumi e Verga si fece addirittura spedire dalla Sicilia stoffe, disegni e vesti usate.

Anche in La lupa abbiamo la violazione di legami familiari sentiti dalla comunità contadina come sacri. Presa da una passione irresistibile, la gnà Pina, detta la Lupa, (l'appellativo animalesco ne colloca la sessualità al di fuori della dimensione umana, nell'ambito degli istinti bestiali), corteggia il giovane Nanni Lasca. Sebbene attratto dalla donna, Nanni pensa innanzi tutto al proprio 'interesse': egli vuole sposarsi in modo da procurarsi una dote e nessuna ragazza lo sposerebbe, se si sapesse di una sua relazione con la matura e vogliosa Lupa. Il giovane sarebbe invece disposto a sposare Mara, la figlia della gnà Pina. Sebbene Mara non voglia sposare Nanni, la madre acconsente al matrimonio diventando poi l'amante del giovane. Passa il tempo Nanni e Mara hanno un figlio e sono felici. La famiglia è quindi la forza salvifica che dà a Nanni la possibilità di redimersi. A questa forza trainante positiva viene opposta l'innaturalità della relazione tra 
Nanni e la gnà Pina. Questa relazione è contro natura e viene 'medicalizzata': apprendiamo che la Lupa si era ammalata e che Nanni era stato addirittura in punto di morte. La guarigione di Nanni viene presentata come un miracolo, frutto delle preghiere alla Madonna da parte di Mara.

Il dramma si conclude il giorno di venerdì santo. Anche qui come nei Mafiusi la punizione dei colpevoli ha le caratteristiche di un intervento divino. Nanni s'è confessato, s'è messo in grazia di Dio; la comunità che lo aveva emarginato lo riaccetta e lo designa addirittura a portare lo stendardo della Confraternita durante la processione. Questo quadro di possibile espiazione viene però turbato dall'arrivo della Lupa che, continuando a desiderare Nanni e non vedendo altra via d'uscita, si fa uccidere da lui.

La motivazione principale che porta alla tragedia finale è la passionalità, ma a questa si aggiunge anche l'interesse economico. Nanni si sposa con Mara per interesse e, una volta sposato, è legato alla 'roba', non riesce ad andare via con Mara in un altro paese per sfuggire alle tentazioni della maliarda gnà Pina. L'emergere della componente economica accanto a quella passionale risulta particolarmente significativo. Siamo nel periodo delle rivolte dei Fasci Siciliani quando con gli scontri sul fronte contadino la Sicilia diventa un epicentro della lotta di classe. Nell'ottica del programma moralistico nazionale di Verga il desiderio di cambiare stato si delinea come un pericoloso elemento perturbatore che minaccia l'equilibrio naturale dell'arcaico' mondo siciliano.

Un'altra opera che nasce nel clima dei Fasci Siciliani è La zolfara di Giuseppe Giusti Sinopoli. La vicenda privata, la storia d'amore, di tradimento e di morte, fra il picconiere Vanni, sua moglie Mara e l'amante di lei, il capomastro Iacopo, si intreccia con l'episodio 'sociale' dello sciopero degli zolfatari che vogliono aumentate le paghe. Il motivo personale, 'caratteriale' e familiare, prevale rispetto alla componente sociale. Non si nega la validità della gerarchia e dei rapporti di potere tra padrone, capomastro e operaio. Piuttosto che uno scontro di classe, si ha una lotta tra buoni e cattivi, tra onesti e disonesti, tra fautori e nemici della famiglia. L'azione si sviluppa dal carattere dei personaggi: Vanni, il marito, è onesto e generoso e cerca in ogni modo di aiutare gli altri picconieri: "l'operaio dabbene", dice alla moglie, "pensa sempre alla famiglia e aiuta il prossimo". Al contrario, Mara e l'amante sono accomunati dalla perfidia e dalla disonestà: Mara imbroglia i clienti della bottega da lei gestita; Iacopo si mette d'accordo con il padrone della zolfara per falsificare cambiali e truffare Vanni.

Concentrando l'attenzione sulla sfera privata e sul carattere, si cerca di depoliticizzare la conflittualità nel Meridione. Le rivolte in Sicilia venivano infatti considerate più una questione di ordine pubblico che di problema- 
tiche sociali. Si diceva che provenissero dallo 'stomaco', dalla povertà di gente semplice e confusa, e non dalla 'testa', cioè da pericolose idee socialiste come le rivolte degli operai del Nord ${ }^{24}$. Come ha notato Anna Barsotti, alla fine la zolfara sarà bruciata da Vanni per vendicarsi del capomastro che gli ha tolto "il pane e l'onore", e non dai picconieri in rivolta ${ }^{25}$. La motivazione privata-caratteriale riduce lo scontro tra il capomastro e l'operaio alla patologia passionale in difesa dell'onore.

In Malia di Luigi Capuana la passione è maleficio demoniaco, sia nella prospettiva corale della comunità, sia in quella individuale della protagonista 'indiavolata', tormentata da struggenti sensi di colpa. Jana viene presa da una passione irresistibile per il cognato Cola. Come la gnà Pina, anche Jana è consapevole di peccare ma è troppo istintiva. Queste donne 'bambine', bloccate in uno stadio infantile, non riescono a controllarsi, avrebbero bisogno di una guida. Pure Cola è bambinesco, i compaesani lo definiscono 'foddi', folle, perchè ha corteggiato e fatto innamorare molte ragazze. L'antidoto alla follia è ovviamente il matrimonio. Il barbiere Taddarita, che combina le nozze tra il giovane e Nedda, sorella di Jana, assicura "Cola però è un bravu giuvini. Lu matrimoniu l'aggiusta" (Cola però è un bravo giovane il matrimonio lo aggiusta) ${ }^{26}$.

Sposarsi segna il passaggio dall'immaturità all'età adulta. Dopo il matrimonio il suocero, rivolgendosi a Cola, sentenzia: "ora si omu daveru" (ora sei uomo davvero) ${ }^{27}$. Il matrimonio è quindi un fatto naturale per chi ha raggiunto la maturità. Chi non si sposa è ancora un bambino, un immaturo. Sposarsi significa anche seguire la volontà divina ed essere responsabili di fronte alle norme religiose della comunità. Taddarita raccomanda: "un sulu Diu e 'na sula mugghieri" (un solo Dio e una sola moglie)28. Il principio religioso non fa che rafforzare la 'naturalità', 'sanità e 'inevitabilità del matrimonio in contrasto con il desiderio peccaminoso di Jana. La passione di Jana spingerà Cola all'adulterio causando la tragedia finale: Nino, il fidanzato di Jana, dopo aver scoperto la tresca di questa con Cola, la perdona e le propone il matrimonio riparatore. Cola si oppone e Nino lo accoltella. Ritroviamo la struttura moralistica con l'ineluttabile punizione dei passionali. La relazione tra Jana e il cognato Cola sconvolge l'ordine naturale (il legame di sangue) e quello religioso (la legge di Dio).

\footnotetext{
24 Dickie, 108.

25 Barsotti, 152.

26 Barbina, 85.

27 Barbina, 100.

28 Barbina, 86.
} 
Il matrimonio è per la comunità contadina qualcosa di naturale e di sacro. Ancora una volta i siciliani sono caratterizzati da sanità morale. Il dubbio che la passione amorosa di Cola e Jana possa essere il risultato di un atto di stregoneria attenua la responsabilità dei peccatori che sono dei siciliani immaturi e istintivi ma fondamentalmente 'onorati'.

\section{La sicilianità come costruzione: il teatro dialettale di Pirandello e la contro- cultura siciliana}

Nel 1909 in un articolo sul teatro siciliano Pirandello individuava il carattere fittizio dell'immagine di una Sicilia 'bestiale', problematizzando così il rapporto tra la Sicilia e il 'fuori della Sicilia':

Ora, fuori dei confini dell'isola, che conoscenza si ha della Sicilia? Una conoscenza limitatissima di poche espressioni caratteristiche, violente, divenute ormai di maniera. Il carattere drammatico siciliano s'è fissato, tipificato nella terribile, meravigliosa bestialità di Giovanni Grasso. Mancando ogni altra conoscenza della vita pur cosí varia e caratteristica della Sicilia, ogn'altra espressione di essa riesce quasi inintelligibile. Non si parli, dunque, di gusti e di tendenze del pubblico; qui si tratta di conoscenza soltanto. Un teatro dialettale, che rappresentasse la vita varia e diversa della Sicilia, potrebbe esser gustato e accolto con fervore solamente in Sicilia: fuori della Sicilia possono aver fortuna soltanto quelle espressioni di cui si ha conoscenza, divenute ormai tipiche; possono aver fortuna cioè il signor Grasso e la signora Aguglia, che non avrebbero neanche bisogno di parlare per farsi applaudire: basterebbe la mimica. Per concludere si vuol creare veramente un teatro dialettale siciliano, o si vuol manifatturare una Sicilia d'esportazione per il signor Grasso e la signora Aguglia?29

Pirandello individua nella produzione del verismo siciliano un importante veicolo di diffusione della tipizzazione 'nazionale' dei siciliani. Questa presa di posizione contro la 'Sicilia d'esportazione' fornisce una chiave di lettura per l'analisi del teatro dialettale pirandelliano in rapporto al discorso nazionale sulla sicilianità. Attraverso l'esame delle strutture drammatiche evidenzieremo il rovesciamento della Sicilia di 'maniera' e l'emergere di un'immagine a-nazionale dell' isola e dei siciliani.

In un articolo del 29 marzo 1918 Antonio Gramsci giudicava Liolà di Pirandello "una delle più belle commedie moderne che la sguaiata critica pseudomoraleggiante ha fatto quasi del tutto ritirare dal repertorio" 30 . A pochi mesi di distanza dalla prima romana al teatro Argentina nel novem-

29 Pirandello, Saggi, poesie, scritti varii, 1208.

30 Gramsci, Letteratura e vita nazionale, 47. 
bre 1916, la commedia era andata in scena al teatro Alfieri di Torino ma, in seguito agli attacchi violentissimi del giornale cattolico Il Momento, era stata tolta dal cartellone. Gramsci, che aveva assistito alla rappresentazione come critico teatrale dell'Avanti, chiarisce le cause dell'insuccesso: "Liolà non finisce secondo gli schemi tradizionali, con una buona coltellata, o con un matrimonio, e perciò non è stata accolta con entusiasmo;(...) per il pubblico ci voleva il sangue o il matrimonio, e perciò il pubblico non ha applaudito" 31 .

In Pirandello la dimensione regionale e dialettale presenta la contestazione dei valori unitari e borghesi sostenuti dal verismo siciliano. Abbiamo il rovesciamento della 'cavalleria rusticana', del mondo 'popolare' e 'arcaico' dell'onore e della fedeltà coniugale borghesi. Il capovolgimento di questi valori si attua attraverso il raddoppiamento parodico di elementi strutturali della produzione verista. Gramsci individuava appunto la mancanza di un finale tradizionale. La sanzione finale svolge un ruolo centrale nella struttura drammatica. Nel teatro verista e in genere in quello borghese la rottura del patto familiare turba l'armonia 'naturale' e innesca la violenza passionale a difesa dell'onore. Proprio con il finale si risolvono le antinomie e si ricompone l'ordine 'naturale' precedentemente sconvolto. La punizione o reintegrazione dei trasgressori adulteri e la gratificazione di coloro che rispettano le regole hanno il carattere di una giustizia trascendente e ribadiscono l'assolutezza dei valori morali presentati sulla scena.

Liolà, il protagonista, è un giovane ardente che di donne 'cento ne vede e cento ne vuole', per cui va in giro ad ingravidare ragazze, prendendo poi con sé i figli nati dalle sue relazioni occasionali. La figura di questo 'impunito' e lucido dongiovanni campagnolo mette in dubbio l'immagine del siciliano passionale e custode della tradizione.

Ma diamo innanzi tutto un'occhiata alla trama. Tuzza, gelosa di Mita, perché questa anche da sposata continua ad essere desiderata da Liolà, si getta per dispetto tra le braccia del giovane e resta incinta. Curiosamente, però, non accetta il matrimonio riparatore che, pure stranamente, un seduttore quale Liolà le offre. Architetta invece un piano piuttosto artificioso e convince don Simuni, marito impotente di Mita, a far passare per suo il figlio di Liolà. Felice di avere un erede a cui lasciare la roba e desideroso di poter mettere a tacere il corale sospetto sulla sua impotenza, don Simuni accusa la moglie, Mita, di essere sterile. A questo punto, però, interviene Liolà. Egli persuade Mita a farsi mettere incinta da lui in modo da 'procurare' anch'ella a don Simuni un figlio. E così avviene. Mita è

31 Gramsci, Letteratura e vita nazionale, 283. 
incinta, don Simuni crede a questa sua paternità legale e vorrebbe ora che Tuzza regolarizzasse la situazione e sposasse Liolà. Il giovane rifiuta il matrimonio ma, come ha già fatto con i tre bambini nati dalle altre relazioni, prenderà con sé il figlio. Tuzza tira fuori il coltello, ma viene fermata da Liolà che conclude la commedia cantando allegramente una canzocina.

Roberto Alonge ha individuato nell'opera passaggi poco credibili e toni fiabeschi ${ }^{32}$. In effetti la commedia è strutturalmente straniante. La tensione, riscontrata da Gramsci nel finale, tra lo schema abituale e lo schema nuovo domina in tutto il corso dell'opera. Invece di identificarsi con i personaggi sentiti come persone reali, il pubblico si concentra sull'azione 'doppia', paragonandola all'azione 'tradizionale' dei personaggi siciliani del teatro verista. Questo contrasto tra azione tradizionale e azione nuova rivela il verismo teatrale e la sicilianità verista come fittizi.

Gli 'ingredienti' del teatro verista sono tutti 'replicati': la passionalità, l'onore, la religione, il matrimonio riparatore, il coltello punitivo. Eppure tali elementi non hanno più la funzione che avevano in quella produzione teatrale. Prendiamo, ad esempio, la passionalità, nel teatro verista questa si sviluppa coerentemente nel corso della commedia come tratto distintivo e realistico del carattere. La passionalità, la mancanza di autocontrollo porta al dramma (Cavalleria rusticana, Malia, La zolfara, La lupa) o innesca un processo di formazione (I Mafiusi). L'azione fecondatrice di Liolà, invece, non provoca nessun dramma d'onore e si presenta piuttosto come 'gestus' parodico, come azione che parodizza la passionalità del carattere siciliano verista.

Anche il canticchiare di Liolà è 'gestus' parodico e richiama l'esprimersi in proverbi e aforismi da parte dei contadini veristi. Il canticchiare non è motivato, non è legato alla vicenda come avveniva per le canzoncine d'amore che la Lupa indirizzava a Nanni o per i proverbi-commenti e favole del saggio coro contadino. Abbiamo, ad esempio, in apertura di La lupa, un racconto contadino su una Maga ammaliatrice che fa cadere gli uomini nel peccato. La figura della Maga ricalca quella della 'gna Pina che nel corso del dramma viene definita, oltre che "lupo mangiatore di uomini" 33 , anche "diavolo di donna" 34 , "diavolo che si fa eremita" 35 , e in chiusura ancora "diavolo" 36 . Il racconto di Filomena sulla Maga preannuncia lo svol-

32 Alonge, Liolà, 11.

33 Verga, Teatro, 283.

34 Verga, Teatro, 291.

35 Verga, Teatro, 287.

36 Verga, Teatro, 324. 
gimento tragico del rapporto peccaminoso tra Nanni e La lupa e rafforza la condanna morale nei confronti dei trasgressori: "La Maga dunque se ne stava nel palazzo incantato, tutto d'oro e di pietre preziose, e come passava un viandante, s'affacciava alla finestra per tirarlo in peccato mortale. Giovani e vecchi, vi cascavano tutti!... religiosi anche, e servi di Dio!..."37. La fiaba viene poi appoggiata da Janu: "Maga o non Maga, sapete come dice il proverbio?: L'uomo è il fuoco, la donna è la stoppa: viene il diavolo e soffia!"38.

Il canterellare di Liolà si distacca da questa morale 'popolare', parodizzando l'immagine della società rurale siciliana come nucleo omogeneo di saggezza atavica:

Zia Croce: Ih, che allegria! Davvero festanti siete! Che miracolo è questo? Liolà: Nessun miracolo, zia Croce. "Chi cerca trova, e chi seguita vince!" Zia Croce: Che vuol dire?

Liolà: Niente. Proverbio ${ }^{39}$.

Il matrimonio riparatore, che dovrebbe concludere la vicenda in lieto fine, qui appare più volte anche nell'antefatto della commedia. Quando apprendiamo che Mita ha rifiutato di sposare Liolà, quest'ultimo ha già tre figli ed è ragazzo-padre, quindi il matrimonio riparatore è stato negato altre tre volte. La moltiplicazione del rifiuto del matrimonio toglie necessità $\mathrm{e}$ sacralità all'istituzione matrimoniale nell'ambito del contesto siciliano.

L'eroe del teatro verista, così come quello del teatro realista in genere, ha un carattere che, nonostante i conflitti, mantiene una sua compattezza. I suoi attributi sono fissi e le sue funzioni stabili. Il personaggio compie un percorso lineare verso il 'bene' attraverso l'espiazione della colpa. Mentre il 'carattere' siciliano verista viene presentato come unitario, passionale e inti mamente buono, rafforzando l'illusione della realtà, Pirandello costruisce il personaggio di Liolà come duplice. Egli è allo stesso tempo buono e cattivo, eroe e antagonista, ripete praticamente lo stesso inganno concepito dalla 'malvagia' Tuzza con l'aggravante dell'adulterio.

Si rompe la tradizionale connessione dell'eroe con le forze positive dei valori morali e di queste con l'elemento religioso. Per convincere Mita a commettere l'adulterio, Liolà assicura che è Dio stesso ad esigere la trasgressione del vincolo matrimoniale in modo da poter punire i due intriganti: "Devi darglielo tu il castigo! Dio stesso te lo comanda!" 40 . Questa

\footnotetext{
37Verga, Teatro, 281.

38 Verga, Teatro, 281.

39 Pirandello, Maschere nude, 932.

40 Pirandello, Maschere nude, 929.
} 
trasgressione legittimata dalla religione viene ribadita da Gesa, zia di Mita, nel primo atto: "neppure una santa del paradiso reggerebbe ai maltrattamenti di questo vecchiaccio, ai raffacci che le fa davanti a tutti. Maria Vergine stessa, vedendosi cimentata così, griderebbe: Ah, tu vuoi davvero un figlio da me? E tieni qua che te lo faccio!" 41.

Il legame tra l'onore siciliano e i valori morali tradizionali viene ancora contestato nel finale con l'apparire del coltello. Nelle opere del teatro verista il coltello punitivo era assegnato ad un personaggio 'onorato', ad Alfio di Cavalleria rusticana o a Nino di Malia, qui invece viene dato a Mita, la quale non ha senso dell'onore, avendo già precedentemente rifiutato il matrimonio riparatore con Liolà.

Come abbiamo rilevato, nel teatro verista la motivazione che dava inizio all'azione era legata alla passione amorosa. L'azione si sviluppava 'naturalmente' dal carattere del personaggio, dando vita ad un dramma 'intimo', familiare. In Pensaci, Giacuminu! (1916) invece il movente rimanda alla sfera sociale. Agostino Toti, professore di scienze, è l'ideatore di una sfida alla società. La sua motivazione è la vendetta contro lo Stato: in età tarda sposa la giovane figlia del bidello, Luzzidda, innamorata di Giacuminu e in attesa di un suo figlio, perché questa usi la pensione dopo la sua morte.

Il gesto del professore rovescia parodicamente il legame tra matrimonio e 'natura' presente nella produzione verista. Il matrimonio tra la Lupa e Nanni è impossibile e 'innaturale', per la differenza di età. Quando la Lupa si dichiara a Nanni il giovane scoppia a ridere: "Voi!... Perché non mi date vostra figlia invece? ...Datemi vostra figlia ch'è carne fresca invece..."42.

Agostino Toti non solo sfida la norma dell'età, ma anche quella della fedeltà coniugale. A proposito delle 'corna', a cui sarebbe destinato sposando una donna giovane, Toti afferma: "Sono nel conto, Sa! Segnate al passivo in precedenza! Ma non per me: se n'andranno in testa alla mia professione di marito, che non mi riguarda se non per l'apparenza. Io anzi vedrò di far tanto che il marito - come marito - le abbia" 43 . Il professore vuole infatti che Luzzidda e Giacuminu continuino a frequentarsi e che allevino insieme il loro bambino. Questo strano triangolo provoca lo scandalo, ma Toti non transige, neanche l'intervento del prete Landolina serve a fargli cambiare idea.

Anche qui, come in Liolà, si rovescia la 'sacralità del patto familiare e si destabilizza il legame verista tra il siciliano e i valori tradizionali della

41 Pirandello, Maschere nude, 913.

42 Verga, Teatro, 296.

43 Pirandello, Maschere nude, 732. 
famiglia e della fede religiosa. La commedia termina con la battuta di Toti diretta contro padre Landolina: "Vade retro! Distruttore della famiglia! Vade retro!". La formula rimanda alla frase (Vangelo di Matteo, cap. IV) con cui Gesù scacciò il diavolo tentatore: "Vade retro Satana!". In questo caso viene invece applicata proprio al consigliere spirituale. Identificando il prete con il diavolo, si mette in dubbio il ruolo della Chiesa come guida morale dell'individuo. Inoltre, il riferimento al Vangelo contro un esponente della Chiesa duplica, sovvertendone la funzione di caratterizzazione della religiosità siciliana, il ricorrere delle immagini religiose nel linguaggio della comunità contadina verista. Infine, la sfida anti-statale di Toti rompe il rapporto 'naturale' del cittadino con lo Stato. Tale rapporto, come abbiamo visto, faceva parte della retorica verista-nazionalista del lavoro che rende felice l'individuo, la famiglia e la nazione (I mafiusi).

In 'A birritta cu' i ciancianeddi (1917) la signora Beatrice rende pubblica la relazione del marito con la moglie dello scrivano Ciampa. Fa una denuncia e fa cogliere in flagrante suo marito con l'amante. I due vengono arrestati e si scatena un vero e proprio tumulto: la madre e il fratello di Beatrice la accusano di non essersi resa conto delle conseguenze disastrose del suo atto che, provocando lo scandalo, ha gettato il disonore su tutta la famiglia. Anche Ciampa accusa la signora Beatrice di avere agito per esercitare la sua vendetta senza pensare a lui, alla sua onorabilità, e di avergli appioppato pubblicamente il cappello a sonagli, il ridicolo cappello da buffone e da 'becco'. Per riacquistare la stima degli altri, pensa Ciampa, bisognerà ammazzare sua moglie e il cavalier Fiorica. Improvvisamente, però, gli balena una soluzione alternativa: occorre che la signora Beatrice risulti pazza, soltanto così si potrà evitare il delitto d'onore.

Ciampa utilizza la sua abilità dialettica e discorsiva per fare passare Beatrice per pazza. La signora Fiorica rifiuta il matrimonio come 'santa' condizione di schiavitù della donna, rinnega l'etica 'verista-siciliana' di sottomissione nei confronti dell'uomo. In un dialogo con la serva Fana, che vuole convincerla a non denunciare il Fiorica, la signora Beatrice, appoggiata dalla rigattiera Saracena, chiarisce il suo desiderio di ribellione al potere del maschio e alla santa pace domestica sostenuta da Ciampa:

LA SARACENA (acchiappando per un braccio Fana e trattenendola): Ah, no no. Aspetti, signora. E anche voi, qua. L'anima l'abbiamo tutti, servi e padroni, davanti a Dio; e non voglio chiacchere, io, sul mio conto. Qual è la coscienza, la vostra, che vedete codesta povera signora pianger lagrime di sangue, patir le pene dell'inferno, e - "Non è niente pazienza l'offra a Dio!" - Questa è la coscienza?

FAna: Questa! Questa! Per chi ha timore di Dio! 
BEATRICE: Ah, e allora un uomo vi tartassa, vi pesta...così...sotto i piedi; è Dio, è vero?

FANA: No. Io dico che dobbiamo offrirlo a Dio, signora mia! Ma quando mai gli uomini, mi scusi, si sono presi così di fronte, a petto? Usar la forza con chi è più forte di noi? Piano piano, signora mia, d'accanto e non di fronte, con garbo e la buona maniera si riportano gli uomini a casa.

LA SARACENA: E già! Mi piace! E per essere così, qua tutte le donne, gli uomini, oh! Toppe da scarpe ne fanno di noi! 44

E più avanti:

FANA: Signora mia, per carità; signora mia, pensi allo scandalo!

BEATRICE: Non me ne importa niente!

FANA: Badi che Vossignoria si rovina!

Beatrice: Mi libero! Mi libero! Mi libero! - Andate Saracena. Non perdiamo più tempo!45

Al contrario, la donna verista accetta e perdona il tradimento. È quanto avviene a Mara la quale si raccomanda alla Madonna affinché le redima Nanni: "Voglio accenderle di tutto cuore le lampade a Maria Addolorata! Tutte le lacrime che ho pianto di nascosto voglio metterle in quelle lampade" 46 . Anche il personaggio di Santuzza in Cavalleria rusticana si assoggetta all'uomo. Santuzza dice di essere disposta a perdonare Turiddu e di riuscire a sopportare da lui tutte le umiliazioni. Quello che fa scattare l'ira, la pazzia di Santuzza, non è il tradimento di Turiddu ma la prepotenza di Lola: "Tu”, dice Santuzza a Turiddu, "puoi camminarmi coi piedi sulla faccia. Ma essa, no!" 47.

Dalla pazzia come 'difetto' del carattere in Cavalleria rusticana si passa qui alla pazzia come costruzione borghese, strumento di controllo e repressione. La pazzia viene attribuita a chi attenta all'ordine sociale e morale borghese. Alla donna gelosa vengono assegnate le caratteristiche della fragilità psichica, dell'isteria. Nella produzione di Verga le didascalie focalizzavano un 'vero intimo' di follia del personaggio, pensiamo al "pallore" e alle "occhiaie scure" della Lupa ${ }^{48}$, allo stesso modo la gestualità prevalente rispetto alla parola doveva rivelare con scientificità lo stato psichico infantile del 'folle'. Santuzza, ad esempio, si copre continuamente il viso con la

\footnotetext{
44 Pirandello, Maschere nude, 770.

45 Pirandello, Maschere nude, 771

46 Verga, Teatro, 307.

47 Verga, Teatro, 222.

48 Verga, Teatro, 278.
} 
mantellina nel corso del dramma. Mentre Santuzza è presentata quale 'naturalmente' isterica, fragile, (e lo stesso si può dire per Jana e la 'gna Pina) a Beatrice il marchio della pazzia viene affibbiato.

Rosario Chiarchiario, il protagonista di 'A patenti (1918), è bollato dalla società col marchio di portasfortuna: è odiato e sfuggito da tutti, perde il posto di lavoro ed è ridotto alla miseria. Invece di negare la calunnia, Chiarchiaro fa ogni sforzo per convalidarla. Egli non solo accetta il ruolo affibbiatogli, ma vuole anche che gli sia rilasciata una patente 'ufficiale' che gli consenta di esercitare legalmente la professione di iettatore. Spaventerà il prossimo e chiederà compensi per non esercitare i suoi oscuri poteri.

Abbiamo la parodia dell'ambiente chiuso, arretrato e immobile della magia meridionale. Pensiamo alla Lupa, alla maliarda, alla donna indiavolata, posseduta, senza possibilità di scampo, dal desiderio peccaminoso e destinata a trascinare inesorabilmente gli uomini nel peccato. Mentre la Lupa subisce passivamente le forze demoniache della passionalità che la portano inesorabilmente alla perdizione e alla punizione finale, Chiarchiaro accetta la posizione di stregone attivamente. La stregoneria non conduce al dramma finale, anzi, attraverso la magia, Chiarchiaro elabora un suo spazio, una sua identità all'interno della comunità. Il giudice settentrionale che deve decidere della questione vorrebbe invece negare l'esistenza della iettatura. Il suo intervento in tal direzione provocherebbe la rovina di Chiarchiaro. Qui è proprio la 'razionalità' del settentrionale a minacciare di innescare la tragedia. Si è portati a riflettere sulla legittimità del giudice che, come dice egli stesso, è chiamato ad "amministrare la giustizia a questi poveri piccoli uomini feroci” 49 . Il rapporto tra meridionali e settentrionali viene problematizzato attraverso la contestazione della visione fatalistica-verista dei meridionali come parte di un universo immobile. Secondo questa visione, che fu poi quella che caratterizzava anche il pensiero di Villari, Sonnino e Franchetti, i meridionali risultavano incapaci di modificare il proprio modo di essere, per cui il cambiamento, eventualmente, doveva venire dal Nord.

Pirandello si muove nell'orbita di quella 'cultura d'opposizione', laica e libertaria, repubblicano-socialista, individuata da Paolo Mario Sipala nella sua analisi della Sicilia del primo Novecento. Dopo aver indicato Napoleone Colajanni come uno tra i principali esponenti di questa 'contro-cultura', Sipala dimostra l'esistenza di un rapporto che legò il sociologo e politico nisseno a Pirandello ${ }^{50}$. Tra il 1906 e il 1907 Pirandello chiese

49 Pirandello, Maschere nude, 891.

50 Paolo Mario Sipala. "Una cosa nuova che la chiamavano sciopero". 
al Colajanni di essere introdotto nell'ambiente radicale e, durante la preparazione del giornale di tendenza anti-clericale La Ragione, il Colajanni raccomandò Pirandello al futuro direttore Arcangelo Ghisleri. Inoltre, proprio uno dei saggi del Colajanni in difesa dei Fasci Siciliani e sugli scandali della Banca romana, Gli avvenimenti in Sicilia e le loro cause (1894), fu utilizzato da Pirandello come fonte privilegiata del suo romanzo storico $I$ vecchi e i giovani (1913) ${ }^{51}$. Il romanzo, come spiega lo stesso Pirandello in una lettera del dicembre 1908 a Ugo Ojetti, doveva rappresentare "il dramma tristissimo della Sicilia dopo il 1870: dramma che si chiude coi fatti del 1893-94". Sono gli anni in cui, con la repressione dei moti siciliani e con gli scandali della Banca Romana, si verifica, continua Pirandello, la "bancarotta del vecchio patriottismo" 52 .

Consapevole delle conseguenze sociali e politiche dell'antropologia criminale, Colajanni fu il principale oppositore delle teorie di Cesare Lombroso, Enrico Ferri e Alfredo Niceforo ${ }^{53}$. All'impostazione razzista

51 La repressione dei Fasci Siciliani è inserita sia da Pirandello che da Colajanni in un contesto italiano fallimentare, un contesto di generale corruzione e malgoverno. Così in un brano dei Vecchi e $i$ giovani viene descritta la delusione per i rapporti tra la Sicilia e il nuovo Regno: "E qual rovinio era sopravvenuto in Sicilia di tutte le illusioni, di tutta la fervida fede, con cui s'era accesa la rivolta! Povera isola, trattata come terra di conquista! Poveri isolani, trattati come barbari che bisognava incivilire! Ed erano calati i Continentali a incivilirli: calate le soldatesche nuove (...); calati tutti gli scarti della burocrazia;(...) e i tribunali militari, e i furti, gli assassinii, le grassazioni, orditi ed eseguiti dalla nuova polizia in nome del Real Governo, e falsificazioni e sottrazioni di documenti e processi politici ignominiosi: tutto il primo governo della Destra parlamentare! E poi era venuta la Sinistra al potere, e aveva cominciato anch'essa con provvedimenti eccezionali per la Sicilia; e usurpazioni e truffe e concussioni e favori scandalosi e scandaloso sperpero di denaro pubblico (...); l'oppressione dei vinti e dei lavoratori, assistita e protetta dalla legge, e assicurata l'impunità agli oppressori...". Pirandello, I vecchi e i giovani, 85-86.

Nel romanzo le proteste e le rivendicazioni dei Fasci dei lavoratori sono considerate come un problema di insufficienza di politiche sociali piuttosto che di ordine pubblico a cui rispondere severamente. Nella visione dei due siciliani i movimenti di rivolta contadina esprimevano delle legittime aspirazioni sociali e, nel quadro dell'insoluta questione agraria, costituivano la reazione del mondo contadino alla miseria e allo sfruttamento.

52 Pirandello, Carteggi inediti, 29.

53 Alfredo Niceforo si ispirò agli studi di Cesare Lombroso sulla devianza e sulla criminalità. In L'Italia barbara contemporanea (1898) e in Italiani del Nord e Italiani del Sud (1901) teorizzò la distinzione tra la razza ariana e la razza mediterranea e quindi l'inferiorità delle popolazioni del Mezzogiorno d'Italia rispetto 
sulla questione meridionale egli oppose una sociologia progressista per cui i problemi del mezzogiorno andavano analizzati, nel quadro di una 'parità razziale', come risultato di insufficienze istituzionali e governative in Sicilia e nel mezzogiorno in genere ${ }^{54}$. L'operazione 'contro-culturale' di Colajanni si svolse mediante la pubblicazione della Rivista popolare di politica, lettere e scienze sociali. I suoi contributi coprono un'area che si estende dalle indagini statistiche ed economiche alle battaglie civili per il divorzio e dalle polemiche antropologiche in difesa dei meridionali alla denuncia dell'assenteismo dello Stato e all'idea di una federalizzazione delle regioni.

Il teatro di Pirandello partecipa alla 'cultura d'opposizione' per l'impostazione laica e per la critica nei confronti della Chiesa come pilastro di moralità, per il rifiuto della concezione dello Stato come unità organica in cui gli individui trovano la loro 'naturale' integrazione collaborando reciprocamente ciascuno secondo la propria 'indole', per la polemica contro i valori borghesi di onore e matrimonio e infine per il rifiuto di una sicilianità come essenza. Il carattere siciliano viene privato dell'attributo fisso ed

a quelle settentrionali. Secondo il Salvadori l'opera di Niceforo: "fu il migliore servigio reso al dominio di classe in Italia, in quanto diede una giustificazione scientifica allo sfruttamento dei contadini meridionali da parte del blocco agrario-industriale, col suo dimostrare eloquentemente che l'umanità si divide in forti e deboli, con tutte le conseguenze che derivano da siffatta teorizzazione". Massimo Salvadori, Il mito del buon governo, 81 .

54 Anche Gramsci segnalerà più tardi l'incomprensione della realtà del meridione e le responsabilità dei positivisti: "la miseria del Mezzogiorno era inspiegabile storicamente per le masse popolari del Nord; esse non capivano che l'unità non era avvenuta su una base di uguaglianza, ma come egemonia del Nord sul Mezzogiorno nel rapporto territoriale di città-campagna, cioè che il Nord concretamente era una piovra che si arricchiva alle spese del Sud e che il suo incremento economico-industriale era in rapporto diretto con l'impoverimento dell'economia e dell'agricoltura meridionale. Il popolano dell'Alta Italia pensava invece che se il Mezzogiorno non progrediva dopo essere stato liberato dalle pastoie che allo sviluppo moderno opponeva il regime borbonico, ciò significava che le cause della miseria non erano esterne, da ricercarsi nelle condizioni economiche-politiche obiettive, ma interne, innate nella popolazione meridionale, tanto più che era radicata la persuasione della grande ricchezza naturale del terreno: non rimaneva che una spiegazione, l'incapacità organica degli uomini, la loro barbarie, la loro inferiorità biologica. Queste opinioni già diffuse (il lazzaronismo napoletano era una leggenda di vecchia data) furono consolidate e addirittura teorizzate dai sociologhi del positivismo (Niceforo, Sergi, Ferri, Orano ecc.) assumendo la forza di "verità scientifica" in un tempo di superstizione per la scienza”. Gramsci, Quaderni del carcere, 9. 
'essenziale' della passionalità e liberato dal legame 'naturale' con gli ambiti di religione e magia. Allo stesso tempo si nega quell'immobilismo congenito della società siciliana che giustificava l'intervento guida da parte del Settentrione.

\section{Independent scholar}

\section{OPERE CITATE}

Alonge, Roberto (a cura di). Liolà. Milano: Mondadori, 1992.

Anderson, Benedict. Imagined Communities: Reflections on the Origin and Spread of Nationalism. London and New York: Verso, 1991.

Barsotti, Anna. Verga drammaturgo. Firenze: La Nuova Italia, 1974.

Barbina, Alfredo (a cura di). Teatro dialettale siciliano. Bologna: Cappelli, 1970.

Bersani, Leo. "Le réalisme et la peur du désir." pp. 47-80 in Barthes, Roland et al. Littérature et réalité. Paris: Éditions du Seuil, 1982.

Bersezio, Vittorio. Le miserie e prosperità del signor Travetti. A cura di Alfredo Jeri. Milano: Biblioteca Universale Rizzoli, 1961.

Bini, Daniela. "Cavalleria Rusticana from Verga and Mascagni to Zeffirelli." Forum Italicum 33. 1(1999): 95-106.

Brecht, Bertolt. Schriften zum Theater, Bd. 3, Frankfurt a. M.: Suhrkamp Verlag, 1963.

Capuana, Luigi. Teatro dialettale siciliano. A cura di Pietro Mazzamuto. Catania: Giannotta, 1974

Ceserani, Remo e Lidia De Federicis. Il Materiale e l'immaginario, vol VII. Torino: Loescher, 1990.

Dickie, John. Darkest Italy. The Nation and Stereotypes of the Mezzogiorno 18601900. Houndmills: Macmillan, 1999.

Duggan, Christopher. Breve storia d'Italia. Casale Monferrato: Piemme, 1998.

Ferrone, Siro. Teatro italiano, V, tomo II. Torino: Einaudi, 1979.

Gramsci, Antonio. Letteratura e vita nazionale. Torino: Einaudi, 1966. Quaderni del carcere. Il Risorgimento. Torino: Einaudi, 1966.

Hobsbawm, Eric. Il trionfo della borghesia: 1848-1875. Bari: Laterza, 2003.

Moe, Nelson. The View from Vesuvius. Italian Culture and the Southern Question. Berkeley: University of California Press, 2002.

Monti, Silvana. Il teatro realista della nuova Italia 1861-1876. Roma: Bulzoni, 1978.

Pirandello, Luigi. Carteggi inediti. A cura di Sarah Zappulla Muscarà. Roma: Bulzoni, 1980.

-. I vecchi e i giovani. A cura di Anna Nozzoli. Milano: Mondadori, 1992.

- Maschere nude. A cura di Italo Borzi e Maria Argenziano. Roma: Newton Compton, 1993.

Saggi, poesie, scritti varii. A cura di Manlio Lo Vecchio-Musti. Milano: Mondadori, 1960. 
Tutto il teatro in dialetto. A cura di Sarah Zappulla Muscarà. Milano: Bompiani, 1993.

Said, Edward. Orientalism. London: Routledge, 1978.

Salvadori, Massimo. Il mito del buon governo. La questione meridionale da Cavour a Gramsci. Torino: Einaudi, 1976.

Sipala, Paolo Mario. "Una cosa nuova che la chiamavano sciopero. Ideologia e letteratura nella Sicilia del primo Novecento.”, pp. 813-860 in Storia d'italia. Le Regioni dall'Unità a oggi. La Sicilia. A cura di M. Aymard e G. Giarrizzo. Torino: Einaudi, 1987.

Szondi, Peter. Teoria del dramma moderno. Torino: Einaudi, 1962.

Verga, Giovanni. Teatro. A cura di Gianni Oliva. Milano: Garzanti, 1987. . Tutte le novelle, vol. II. Milano: Mondadori, 1963.

Zappulla Enzo (a cura di). Angelo Musco e il teatro del suo tempo. Catania: Maimone editore, 1991.

"Giovanni Grasso, magnifico attore tragico siciliano." pp. 347-357 in L'Europa che comincia e finisce: la Sicilia. A cura di Dagmar Reichardt. Frankfurt am Main, 2006.

Zappulla Muscarà, Sarah. Introduzione. Tutto il teatro in dialetto. Luigi Pirandello. Milano: Bompiani, 1993. v-xxxii. 